\title{
A PROOF OF HILBERT'S THEOREM ON TERNARY QUARTIC FORMS
}

\author{
JiA XU AND YONG YAO
}

Abstract. Hilbert's theorem states that every positive semi-definite real ternary quartic form can be written as a sum of squares of quadratic forms. In this paper, a constructive proof based on the method called ladder technique is presented. A practical example is proposed to illustrate that this method can be used to prove some hard inequalities on ternary quartic forms.

Mathematics subject classification (2010): 11E25, 14P05.

Keywords and phrases: Hilbert's theorem, positive semi-definite, ternary quartic forms, sum of squares, the ladder technique.

\section{REFERENCES}

[1] Canny J., Manocha D., Multipolynomial resultant algorithms, J. symbolic Comput., 15, 2 (1993) 99-122.

[2] Choi M. D., Lam T. Y., Extremal positive semidefinite forms, Math. Ann. 231, (1977), 1-26.

[3] Choi M. D., LAm T. Y., ReZnick B., Sums of squares of real polynomials, Symp. in Pure Math 58, (1995) 103-126.

[4] Cirtoaje V., Algebraic inequalities, old and new methods, GIL Publishing House, Zalău, Romania (2006) 77-78, 70-72.

[5] Hilbert D., Über die Darstellung definiter Formen als Summe von Formenquadraten, Math. Ann., 32, (1888), 342-350.

[6] Kapur D., SAXena T., YAng L., Algebraic and Geometric reasoning using Dixon resultants, Proc. ISSAC (1994) 99-107.

[7] PARrilo P. A., Semidefinite programming relaxations for semialgebraic problems, Mathematical Programming 96, (2003) 293-320.

[8] PFister A., SCHEIDERER C., An elementary proof of Hilbert's theorem on ternary quartics, Journal of Algebra 371, (2012), 1-25.

[9] Powers V., Reznick B., Scheiderer C., Sottile F., A new approach to Hilbert's theorem on ternary quartics, C. R. Acad. Sci. Paris, Sur. I 339, (2004) 617-620.

[10] Rouillier F., Solving Zero-Dimensional Systems through the Rational Univariate Representation, Applicable Algebra in Engineering, Communication and Computing 9, 5 (1999) 433-461.

[11] SCHEIDERER C., Hilbert's theorem on positive ternary quartics: A refined analysis, J. Algebraic Geom. 19, (2010) 285-333.

[12] Xia B. C., YANG L., Automated inequality proving and discovering, World Scientific Publishing Co. Pte. Ltd, Singapore (2016) 109-125, 182-229.

[13] YANG L., XIA S. H., An inequality proving program applied to global optimization, In W. C. Yang et al (eds.), Proceedings of ATCM 2000, Blacksbug: ATCM, Inc. (2000) 40-51. 